\title{
DIÓXIDO DE TITÂNIO SOL-GEL: PROPRIEDADES E COMPORTAMENTO ELETROCRÔMICO
}

\author{
Rita Aparecida Zoppi* e Nelson Henrique Ribeiro Mortean
}

Instituto de Ciências Biológicas e Química - Pontifícia Universidade Católica de Campinas - CP 1111 - 13020-904 - Campinas - SP

Recebido em 23/8/99; aceito em 19/5/00

\begin{abstract}
SOL-GEL TITANIUM DIOXIDE: PROPERTIES AND ELECTROCHROMIC BEHAVIOR. Titanium dioxide was prepared by hydrolysis and polycondensation of titanium tetraisopropoxide. $\mathrm{TiO}_{2}$ films were obtained by spin coating of the precursor solution on ITO substractes (glass covered with indium doped tin oxide). Films were prepared using different temperatures and hydrochloric acid contents. The effect of the drying temperature of the films $\left(100\right.$ or $\left.400^{\circ} \mathrm{C}\right)$ was also investigated. $\mathrm{TiO}_{2}$ films were characterized by cyclic voltammetry, chronoamperometry, ultraviolete-visible spectroscopy, scanning electron microscopy and $\mathrm{X}$-ray diffractrometry.
\end{abstract}

Keywords: sol-gel process; $\mathrm{TiO}_{2}$; electrochemical properties.

\section{INTRODUÇÃO}

Filmes finos de óxidos de metais de transição têm sido preparados pelo processo sol-gel sobre diferentes tipos de substratos. Suas propriedades eletroquímicas ${ }^{1-3}$ e suas potencialidades de aplicação como sensores ${ }^{4-10}$, em catálise ${ }^{11-12}$, cátodos em células recarregáveis a base de lítio e eletrólitos poliméricos ${ }^{13}$, e fotocatalisadores em processos de degradação de poluentes orgânicos ${ }^{14-16}$, têm sido recentemente investigadas. O processo sol-gel tem sido usado ainda para preparar óxidos mistos tais como $\mathrm{ZrO}_{2}-\mathrm{SiO}_{2}{ }^{17-21}, \mathrm{ZrO}_{2}-\mathrm{TiO}_{2}{ }^{21-23}, \mathrm{ZrO}_{2}-\mathrm{WO}_{3}{ }^{24}, \mathrm{ZrO}_{2}-$ $\mathrm{Al}_{2} \mathrm{O}_{3}{ }^{25}$ e $\mathrm{ZrO}_{2}-\mathrm{Y}_{2} \mathrm{O}_{3}{ }^{26-28}, \mathrm{TiO}_{2}-\mathrm{SiO}_{2}{ }^{29-30}, \mathrm{TiO}_{2}-\mathrm{CeO}_{2}{ }^{31-33}, \mathrm{TiO}_{2^{-}}$ $\mathrm{IrO}_{2}{ }^{31-34}, \mathrm{TiO}_{2}-\mathrm{Fe}_{2} \mathrm{O}_{3}{ }^{1}$ e $\mathrm{TiO}_{2}-\mathrm{RuO}_{2}{ }^{35}$.

Eletrodos de grandes dimensões, numa escala industrial de produção de janelas eletrocrômicas, já são fabricados usandose a técnica de "dip-coating". Camadas de $\mathrm{WO}_{3}$ podem ser preparadas facilmente pelo processo sol-gel e têm mostrado propriedades eletrocrômicas similares às obtidas pelas técnicas convencionais.

Ainda devido às propriedades eletrocrômicas apresentadas pelos óxidos metálicos, a produção de espelhos eletrocrômicos constitui um outro mercado. O espelho é constituído de um eletrodo condutor transparente (por exemplo ITO) coberto com um filme de material de coloração anódica (por exemplo óxido de níquel ou irídio), seguido de um eletrólito sólido (pentóxido de tântalo) e um filme de coloração catódica (por exemplo, $\mathrm{WO}_{3}$ ) depositado sobre um eletrodo condutor refletor (geralmente um filme de alumínio ou platina). Os espelhos retrovisores dos automóveis mais avançados no momento são eletrocrômicos. A refletância destes espelhos é da ordem de $50 \%$ para o estado claro e $15 \%$ para o estado escuro. O tempo de resposta varia de 2 a 4 segundos e sua durabilidade é de aproximadamente $10^{7}$ ciclos de clareamento e escurecimento ${ }^{36}$.

Muitos óxidos de metal de transição podem ser preparados via sol-gel, porém seu comportamento eletrocrômico tem sido pouco investigado ${ }^{37} \cdot \mathrm{WO}_{3} \cdot \mathrm{nH}_{2} \mathrm{O}$ amorfo, filmes de $\mathrm{TiO}_{2}$ ou $\mathrm{V}_{2} \mathrm{O}_{5}$, têm sido obtidos a partir de etóxido de tungstênio, Ti $\left[\mathrm{O}\left(\mathrm{CH}_{2}\right)_{3} \mathrm{CH}_{3}\right]_{4}$ e ácido polivanádico, respectivamente. A síntese de materiais eletrocrômicos pelo processo sol-gel oferece algumas vantagens ${ }^{37}$ :

1) tem sido observado que as propriedades eletrocrômicas do $\mathrm{WO}_{3}$ são muito sensíveis ao método de preparação. Filmes obtidos por "sputtering" apresentam tempos de resposta

e-mail: labpol@acad.puccamp.br mais curtos que os obtidos por evaporação à vácuo. Isto tem sido relacionado ao conteúdo de água no material. Filmes mais hidratados apresentam melhor reversibilidade e tempos de resposta mais curtos. Usando-se o processo solgel, os filmes sempre contêm água e apresentam estrutura porosa, facilitando a difusão de íons e melhorando a performance do material;

2) há a possibilidade de preparação de dispositivos do tipo gel multi-camada. Todas as camadas ativas do dispositivo (eletrólitos e materiais eletrocrômicos) podem ser obtidas a partir de géis. Tais sistemas exibem tempo de resposta longo, boa durabilidade à ciclagem e memória óptica muito longa, abrindo caminho para novos dispositivos micro-iônicos ${ }^{38}$.

Neste trabalho, filmes de $\mathrm{TiO}_{2}$ foram preparados a partir da hidrólise e condensação do tetraisopropóxido de titânio. $\mathrm{O}$ efeito das condições de preparação dos sóis e da temperatura de secagem dos filmes sobre as propriedades do material final foi investigado.

\section{PARTE EXPERIMENTAL}

\section{Preparação do óxido semicondutor}

Os filmes de óxido de titânio foram obtidos a partir de sóis preparados como segue:

$1,20 \mathrm{~mL}$ de tetraisopropóxido de titânio (Aldrich), TiOP, foi diluído em $30 \mathrm{~mL}$ de álcool isopropílico P.A. (Merck). Adicionou-se à mistura diferentes volumes $(50,100,200 \mathrm{ou} 400 \mu \mathrm{L})$ de $\mathrm{HCl}$ concentrado (Merck).

A mistura foi agitada por $1 \mathrm{~h}$ em frasco aberto, a diferentes temperaturas $\left(0,5,25\right.$ ou $\left.50^{\circ} \mathrm{C}\right)$. Os sóis $(2,5 \mathrm{~mL}-$ adicionados de 100 em $100 \mu \mathrm{L}$ ) foram depositados sobre eletrodos de ITO (área $=5 \mathrm{~cm}^{2}$ ) por "spin-coating" a 3000rpm em um Photoresist Spinner Headway Research EC101DT-R485. As amostras foram tratadas durante $1 \mathrm{~h}$ a uma temperatura de $100^{\circ} \mathrm{C}$ ou de $400^{\circ} \mathrm{C}$.

\section{Caracterização do óxido semicondutor}

As propriedades eletroquímicas dos filmes foram investigadas por voltametria cíclica e cronoamperometria. A voltametria cíclica foi feita na faixa de $-2,0$ a $0,3 \mathrm{~V}$ a diferentes velocidades de varredura $(5,10,15,20,25,35 \mathrm{e} 45 \mathrm{mV} / \mathrm{s})$, e as medidas de cronoamperometria foram realizadas aplicando-se pulsos de potencial de 0 a $-2,0 \mathrm{~V}$, com tempo de pulso que variou de 5 a $15 \mathrm{~s}$. As medidas foram feitas em um potenciostato/galvanostato 
AUTOLAB ECO CHEMIE - PGSTAT20. O eletrólito utilizado foi o perclorato de lítio (Aldrich) em carbonato de propileno (Aldrich) destilado, solução $1 \mathrm{~mol} \cdot \mathrm{dm}^{-3}$. Foi utilizada uma cela eletroquímica com 3 eletrodos, tendo como contra eletrodo uma placa de platina e um eletrodo de prata/cloreto de prata de dupla junção como eletrodo de referência.

As propriedades eletrocrômicas dos filmes foram investigadas por espectroscopia no ultravioleta-visível. A cela eletroquímica foi instalada em um espectrofotômetro Hewlett Packard 8453 e espectros na faixa de 350 a $1000 \mathrm{~nm}$ foram registrados polarizando-se os filmes em diferentes potenciais.

Os filmes foram caracterizados também por difratometria de raios-X em um difratômetro XD-3A Shimadzu utilizando radiação do cobre $\mathrm{K} \alpha$. Microscopia eletrônica de varredura foi feita em um microscópio Jeol JS-T300, a um potencial de 20kV. As amostras foram metalizadas com ouro por "sputtering".

A espessura dos filmes foi medida em um perfilômetro Alpha Step 200 Tencor Instruments.

\section{RESULTADOS E DISCUSSÃO}

\section{Estudos preliminares da preparação dos sóis}

Na Figura 1 são mostrados os voltamogramas cíclicos de filmes de $\mathrm{TiO}_{2}\left(100^{\circ} \mathrm{C}\right)$ obtidos a partir de sóis recém-preparados e sóis envelhecidos por 1 mês. Sóis recém-preparados correspondem a sóis preparados e depositados no mesmo dia para a obtenção do óxido.

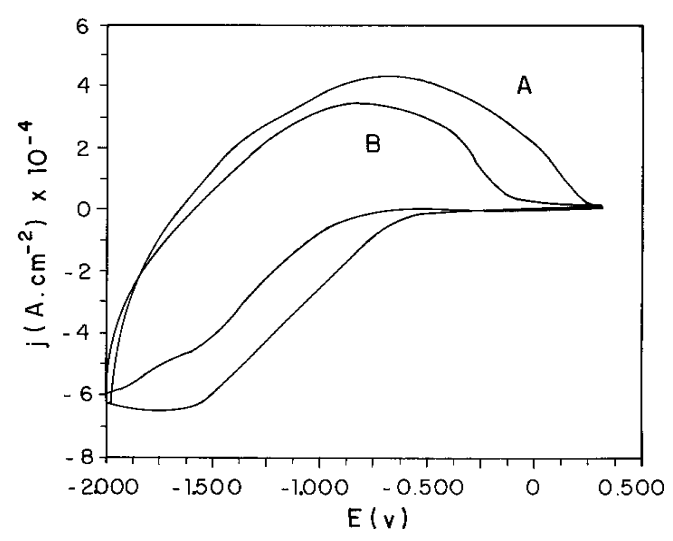

Figura 1. Voltametria cíclica de filmes de $\mathrm{TiO}_{2}$ preparados a partir de sóis (a) recém-preparados e (b) sóis envelhecidos por 1 mês. Temperatura de secagem dos sóis $($ Tsec $)=100^{\circ} \mathrm{C}$. Volume de $\mathrm{HCl}=50 \mu \mathrm{L}$.

Os voltamogramas mostrados na Figura 1 são muito similares aos mostrados na literatura para o óxido de titânio amorfo ${ }^{37}$. O óxido de titânio é classificado como material eletrocrômico de coloração catódica e a reação de intercalação que ocorre durante o processo redox é dada abaixo:

$$
\begin{array}{cl}
\mathrm{TiO}_{2}+\mathrm{Li}^{+}+\mathrm{e}^{-} / & \mathrm{TiOOLi} \\
\text { (transparente - oxidado) } & \text { (cinza-azulado - reduzido) }
\end{array}
$$

A variação de cor foi registrada simultaneamente à ciclagem do potencial entre $-2,0$ a $0,3 \mathrm{~V}$, Figura 2. Verificase que no extremo anódico o filme é transparente e a absorbância é próxima de zero na faixa de 350 a 1000nm. Com a diminuição do potencial aplicado ao filme, o espectro passa a apresentar uma larga banda de absorção com intensidade máxima próxima de $800 \mathrm{~nm}$, mostrando que o filme encontrase no estado colorido, no caso cinza-azulado. São observadas bandas menos intensas com máximos de absorção próximos de 400 e $500 \mathrm{~nm}$.

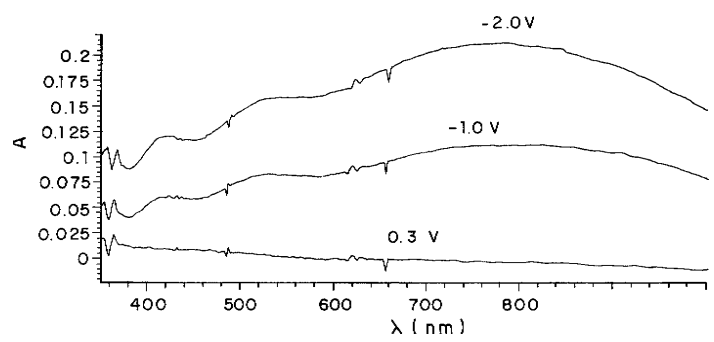

Figura 2. Espectros na região do ultravioleta-visível de filmes de $\mathrm{TiO}_{2}$ preparados a partir de sóis recém-preparados. Filmes polarizados a $0,3,-1,0$ e $-2,0 \mathrm{~V}$. Tsec $=100^{\circ} \mathrm{C}$. Volume de $\mathrm{HCl}=50 \mu \mathrm{L}$.

Para os filmes obtidos a partir dos sóis envelhecidos durante 1 mês, o voltamograma praticamente não apresenta alterações significativas. Neste caso, os espectros na região do ultravioleta-visível foram similares aos mostrados na Figura 2.

Foram preparados também filmes a partir de sóis envelhecidos durante dois meses. Embora os voltamogramas tenham sido similares aos mostrados na Figura 1, a variação de absorbância entre o estado claro e o estado escuro foi praticamente duas vezes menor comparada aos filmes preparados a partir dos sóis envelhecidos durante 1 mês. Comparando-se com sóis recém-preparados, a variação de absorbância nos filmes obtidos dos sóis envelhecidos por 2 meses foi praticamente 5 vezes menor. Isto mostra que o tempo de envelhecimento dos sóis influencia drasticamente as propriedades eletroquímicas e eletrocrômicas do óxido.

Na Figura 3 é mostrado o voltamograma cíclico de filmes de $\mathrm{TiO}_{2}$ obtidos a partir de sóis recém-preparados e que foram secos a uma temperatura de $400^{\circ} \mathrm{C}$. Novamente, as propriedades eletroquímicas e eletrocrômicas são distintas se estes são comparados aos filmes obtidos a partir de sóis secos a $100^{\circ} \mathrm{C}$. O voltamograma apresenta dois picos anódicos em aproximadamente $-1,2$ e $-0,5 \mathrm{~V}$, e o espectro de absorção apresenta bandas mais definidas próximas de 400 e 500nm, além da banda larga em 800nm, Figura 4.

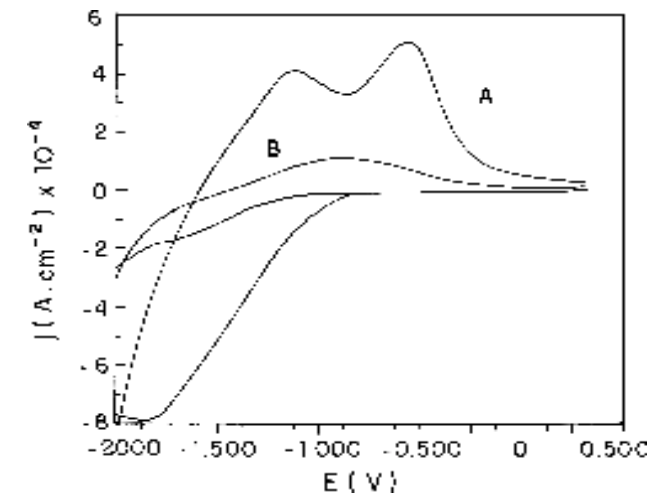

Figura 3. Voltametria cíclica de filmes de $\mathrm{TiO}_{2}$ preparados a partir de sóis recém-preparados. (a) Tsec $=400^{\circ} \mathrm{C}$ e volume de $\mathrm{HCl}=50 \mu \mathrm{L}$. (b) $\mathrm{Tsec}=100^{\circ} \mathrm{C}$ e volume de $\mathrm{HCl}=100 \mu \mathrm{L}$.

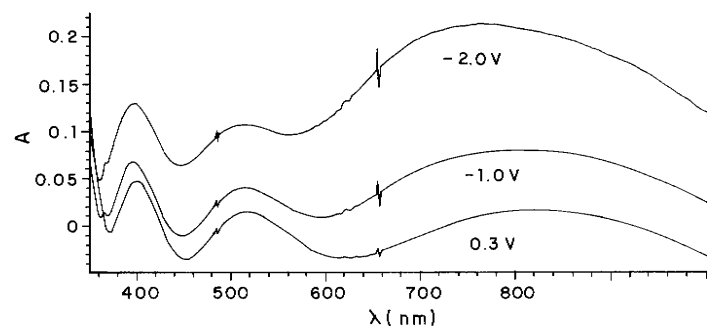

Figura 4. Espectros na região do ultravioleta-visível de filmes de $\mathrm{TiO}_{2}$ preparados a partir de sóis recém-preparados. Filmes polarizados a $0,3,-1,0$ e $-2,0 \mathrm{~V}$. Tsec $=400^{\circ} \mathrm{C}$. Volume de $\mathrm{HCl}=50 \mu \mathrm{L}$. 
Para os sóis envelhecidos durante 1 mês, o comportamento foi similar ao observado para os filmes obtidos de sóis recémpreparados. Já os filmes obtidos de sóis envelhecidos durante 2 meses praticamente não apresentaram variação no espectro de absorção em função do potencial aplicado ao filme.

$\mathrm{O}$ fato de serem observados picos anódicos melhor definidos para os materiais obtidos após a secagem a $400^{\circ} \mathrm{C}$, deve estar relacionado a mudanças estruturais. Em filmes de óxido de titânio amorfo, a inserção de $\mathrm{Li}^{+}$ocorre aleatoriamente, e não são observados picos no voltamograma de tais filmes. $\mathrm{O}$ $\mathrm{TiO}_{2}$ amorfo cristaliza na forma anatase por aquecimento a $400^{\circ} \mathrm{C}$. A fase rutilo é obtida por aquecimento a temperaturas mais altas, ou mesmo por tratamento térmico a $400^{\circ} \mathrm{C}$ por tempos prolongados ${ }^{37}$.

\section{Efeito da temperatura e do volume de ácido usados na preparação dos sóis}

Sóis foram preparados a partir da mistura de TiOP/ isopropanol/ $\mathrm{HCl}(50 \mu \mathrm{L})$ em duas temperaturas diferentes: a $0,5^{\circ} \mathrm{C}$ e a $50^{\circ} \mathrm{C}$. Durante o depósito, os sóis foram mantidos nas respectivas temperaturas. A secagem dos sóis nestes casos foi feita a $100^{\circ} \mathrm{C}$.

Os filmes obtidos a partir dos sóis preparados a $0,5^{\circ} \mathrm{C}$ não puderam ser caracterizados. Neste caso, após a secagem dos sóis, os filmes adquiriram coloração rosada, apresentaram trincas e desprenderam do eletrodo quando este foi imerso no eletrólito.

Filmes obtidos a partir de sóis preparados a $50^{\circ} \mathrm{C}$ mostraram voltamogramas e espectros de absorção similares aos das Figuras 1a e 2, respectivamente, evidenciando que a temperatura de preparação, Tprep, dos sóis deve ser controlada. Para temperaturas abaixo de $20^{\circ} \mathrm{C}$, não se consegue depositar filmes com boas propriedades de adesão ao eletrodo de ITO.

$\mathrm{Na}$ Figura $3 \mathrm{~b}$ é mostrado o voltamograma cíclico de filmes que foram obtidos a partir de sóis preparados a $50^{\circ} \mathrm{C}$, porém o volume de $\mathrm{HCl}$ utilizado foi igual a $100 \mu \mathrm{L}$. O comportamento dos filmes obtidos a partir de sóis preparados com 200 e $400 \mu \mathrm{L}$ de $\mathrm{HCl}$ foi muito similar ao mostrado na Figura $3 \mathrm{~b}$. A intensidade das correntes anódica e catódica foi significativamente menor comparada ao voltamograma da Figura 1a, indicando que um excesso de ácido pode inibir de forma exagerada a hidrólise e condensação do precursor, não ocorrendo a formação do óxido. O espectro de absorção também mostrou que apenas uma quantidade muito pequena de material eletroativo estava presente no eletrodo, pois a variação de absorbância entre o estado claro e o estado escuro, em $800 \mathrm{~nm}$, foi próxima de 0,025 .

Estes resultados mostram claramente que as propriedades eletroquímicas e eletrocrômicas do material depositado sobre o eletrodo dependem fortemente das condições de preparação dos sóis precursores, que devem ser rigorosamente controladas. Para obter filmes com propriedades eletrocrômicas satisfatórias, os sóis devem ser preparados a temperaturas superiores a $25^{\circ} \mathrm{C}$, deve-se usar um volume de ácido suficiente apenas para evitar a precipitação imediata do óxido e os sóis devem ser utilizados logo após a sua preparação (sóis recém-preparados).

\section{Voltametria cíclica em diferentes velocidades de varredura}

Os voltamogramas cíclicos dos filmes preparados a 100 e a $400^{\circ} \mathrm{C}$ feitos em diferentes velocidades de varredura são mostrados na Figura 5. Em ambos os casos, notou-se um aumento da corrente de pico $\left(i_{p}\right)$ com o aumento da velocidade de varredura. Simultaneamente foi observado um deslocamento dos potenciais de pico $\left(\mathrm{E}_{\mathrm{p}}\right)$, sendo o pico anódico deslocado para potenciais mais anódicos e o pico catódico para potenciais mais catódicos. Valores maiores de correntes de pico foram observados para os filmes preparados a $100^{\circ} \mathrm{C}$. Como a quantidade de sol depositada nos eletrodos foi a mesma, e portanto a quantidade de material eletroativo presente na superfície do eletrodo deve ser a mesma, acredita-se que as diferenças observadas sejam devidas efetivamente à eletroatividade dos filmes e não à quantidade de material presente. Observa-se ainda que a separação entre os potenciais de pico anódico e catódico é menor no caso do filme seco a $100^{\circ} \mathrm{C}$ comparada ao filme preparado a $400^{\circ} \mathrm{C}$, o que sugere que o último é menos eletroativo.
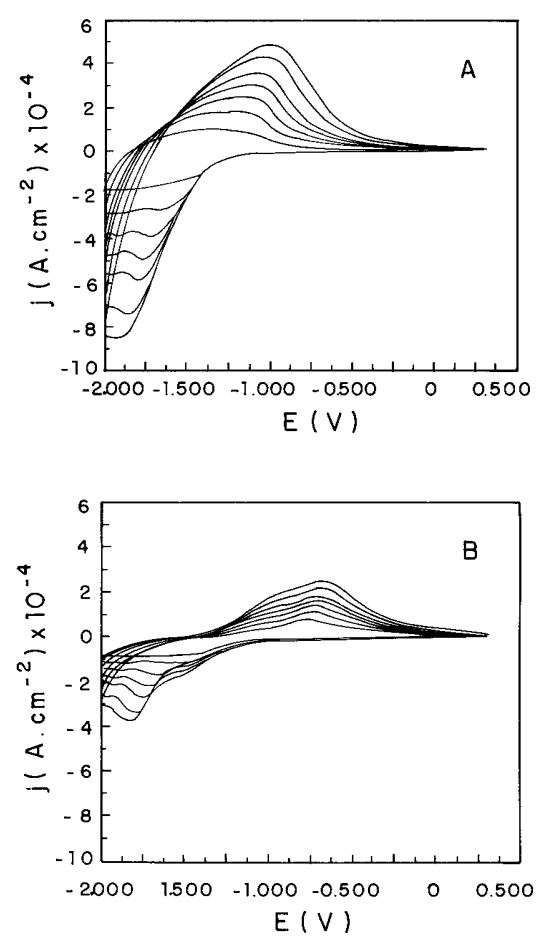

Figura 5. Voltametria cíclica em diferentes velocidades de varredura $(5,10,15,20,25,35$ e $45 \mathrm{mV} / \mathrm{s})$ de filmes de $\mathrm{TiO}_{2}$ preparados a partir de sóis recém-preparados (Tprep $=50^{\circ} \mathrm{C}$ e volume de $\left.\mathrm{HCl}=50 \mu \mathrm{L}\right)$. (a) $\mathrm{Tsec}=100^{\circ} \mathrm{C}$. (b) $\mathrm{Tsec}=400^{\circ} \mathrm{C}$.

Conforme mostrado na Figura 6, quando se analisa o comportamento da corrente de pico, $\mathrm{i}_{\mathrm{p}}$, como uma função da velocidade de varredura, $v$, observa-se que $i_{p}$ varia linearmente com a raiz quadrada da velocidade de varredura. Este mesmo comportamento foi observado para os filmes secos a $100^{\circ} \mathrm{C}$, sugerindo que em ambos os casos o transporte de massa ocorre através de uma difusão linear semi-infinita. Assim, admitindose que o comportamento redox do $\mathrm{TiO}_{2}$ é ideal, pode-se estimar um coeficiente de difusão para os íons intercalantes $\left(\mathrm{Li}^{+}\right)$, utilizando o valor do coeficiente angular do gráfico de $\mathrm{i}_{\mathrm{p}}$ versus $\mathrm{v}^{1 / 2}$. O coeficiente angular obtido para os filmes secos a $100^{\circ} \mathrm{C}$ foi o dobro $(0,0004)$ daquele observado para os filmes secos a $400^{\circ} \mathrm{C}(0,0002)$. Este coeficiente angular é diretamente proporcional ao coeficiente de difusão dos contra íons. Os valores encontrados mostram que o transporte de massa é mais rápido nos filmes de $\mathrm{TiO}_{2}$ preparados a $100^{\circ} \mathrm{C}$.

Outra informação possível de ser obtida dos voltamogramas diz respeito à reversibilidade do sistema. A razão entre as cargas anódica e catódica $\left(\mathrm{q}_{\mathrm{a}} / \mathrm{q}_{\mathrm{c}}\right)$ é um parâmetro usado para obter informações sobre a reversibilidade das transições redox. Para um sistema reversível, $\mathrm{q}_{\mathrm{a}} / \mathrm{q}_{\mathrm{c}}$ deve ser igual a 1 , independente da velocidade de varredura. Tanto para o filme seco a $100^{\circ} \mathrm{C}$ como para o filme seco a $400^{\circ} \mathrm{C}, \mathrm{q}_{\mathrm{a}} / \mathrm{q}_{\mathrm{c}}$ aumentou com o aumento da velocidade de varredura praticamente na mesma proporção, ou seja, usando-se este parâmetro para testar a reversibilidade, a resposta dos filmes é semelhante. Um outro parâmetro geralmente utilizado é a diferença entre o potencial de 
Tabela 1. Potenciais de pico anódico e catódico, $\Delta \mathrm{E}_{\mathrm{p}}\left(\mathrm{Ep}_{\mathrm{a}}-\mathrm{Ep}_{\mathrm{c}} ; \mathrm{V}\right)$ e razão entre as cargas anódica e catódica ( $\left.\mathrm{q}_{\mathrm{a}} / \mathrm{q}_{\mathrm{c}}\right)$ em função da velocidade de varredura $(\mathrm{v} ; \mathrm{mV} / \mathrm{s})$ observados para filmes de $\mathrm{TiO}_{2}$ secos a 100 e a $400^{\circ} \mathrm{C}$.

\begin{tabular}{ccccccccc}
\hline \multicolumn{3}{c}{$100^{\circ} \mathrm{C}$} & \multicolumn{3}{c}{$400^{\circ} \mathrm{C}$} \\
\hline $\mathrm{v}$ & anódico & catódico & $\mathrm{Ep}_{\mathrm{a}}-\mathrm{Ep}_{\mathrm{c}}$ & $\mathrm{q}_{\mathrm{a}} / \mathrm{q}_{\mathrm{c}}$ & anódico & catódico & $\mathrm{Ep}_{\mathrm{a}}-\mathrm{Ep}_{\mathrm{c}}$ & $\mathrm{q}_{\mathrm{a}} / \mathrm{q}_{\mathrm{c}}$ \\
5 & $-1,434$ & $-1,477$ & 0,043 & 0,81 & $-0,789$ & $-1,434$ & 0,645 & 0,98 \\
10 & $-1,198$ & $-1,686$ & 0,488 & 0,98 & $-0,754$ & $-1,625$ & 0,871 & 1,12 \\
15 & $-1,164$ & $-1,738$ & 0,574 & 1,14 & $-0,728$ & $-1,677$ & 0,949 & 1,17 \\
20 & $-1,12$ & $-1,765$ & 0,645 & 1,17 & $-0,711$ & $-1,73$ & 1,019 & 1,18 \\
25 & $-1,094$ & $-1,8$ & 0,706 & 1,28 & $-0,711$ & $-1,756$ & 1,045 & 1,18 \\
35 & $-1,05$ & $-1,869$ & 0,819 & 1,43 & $-0,685$ & $-1,8$ & 1,115 & 1,24 \\
45 & $-1,016$ & $-1,921$ & 0,905 & 1,66 & $-0,676$ & $-1,834$ & 1,158 & 1,52 \\
\hline
\end{tabular}

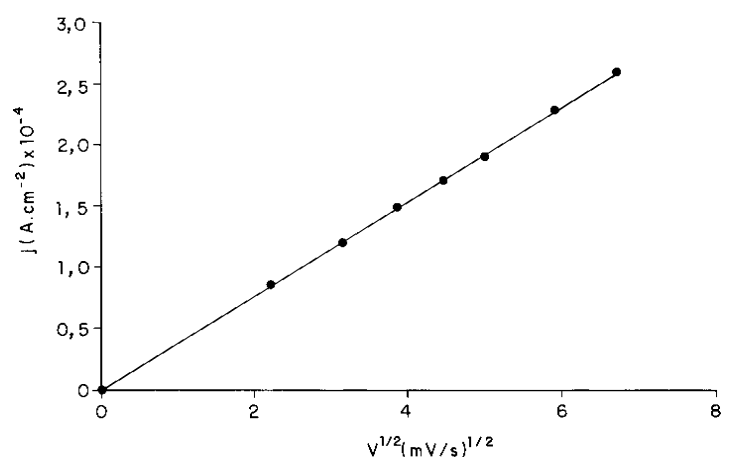

Figura 6. Corrente de pico anódica em função da raiz quadrada da velocidade de varredura de filmes de $\mathrm{TiO}_{2}$ preparados a partir de sóis recém-preparados. Tprep $=50^{\circ} \mathrm{C}$, volume de $\mathrm{HCl}=50 \mu \mathrm{L}, e$ Tsec $=400^{\circ} \mathrm{C}$.

pico anódico e catódico, $\Delta \mathrm{E}_{\mathrm{p}}$. Se $\Delta \mathrm{E}_{\mathrm{p}}$ for maior que $0,9 \mathrm{~V}$, temse um sistema irreversível. Isto significa que nem todos os contra íons que entraram no filme ocupando os sítios eletroativos durante o processo de intercalação, saíram durante o processo de deintercalação. Na Tabela 1 são mostrados os valores de potencial de pico anódico e catódico apresentados pelos filmes de $\mathrm{TiO}_{2}$ preparados a $100^{\circ} \mathrm{C}$ e a $400^{\circ} \mathrm{C}$. Com o aumento da velocidade de varredura há um aumento de $\Delta \mathrm{E}_{\mathrm{p}}$. Para os filmes preparados a $400^{\circ} \mathrm{C}$, são atingidos valores maiores que $0,9 \mathrm{~V}$ mesmo quando a velocidade de varredura é baixa $(15 \mathrm{mV} / \mathrm{s})$.

Para verificar se os filmes preparados a $400^{\circ} \mathrm{C}$ tendem a um comportamento efetivamente irreversível, foram construídos gráficos de potencial de pico em função da corrente de pico. $\mathrm{O}$ coeficiente angular de tais curvas fornece os valores da resistência relativa à queda ôhmica $(\mathrm{R})$. Nestes casos, os valores de $\mathrm{R}$ foram 610 e $540 \mathrm{ohm} . \mathrm{cm}^{2}$ para os filmes secos a $100^{\circ} \mathrm{C}$ e a $400^{\circ} \mathrm{C}$, respectivamente, ou seja, foram da mesma ordem de grandeza. Como os valores de $\mathrm{R}$ são próximos, o comportamento apresentado pelo filme seco a $400^{\circ} \mathrm{C}$ parece ser mesmo característico de um sistema irreversível e de um material menos eletroativo que aquele preparado a $100^{\circ} \mathrm{C}$. Além disso, os resultados de $\mathrm{R}$ são coerentes considerando-se os valores de espessura dos filmes que foram da ordem de 130 e $30 \mathrm{~nm}$ para os filmes secos a 100 e a $400^{\circ} \mathrm{C}$, respectivamente.

\section{Cronoamperometria}

A partir dos dados de cronoamperometria foi possível obter informações a respeito da estabilidade do filme com a ciclagem do estado oxidado ao reduzido e vice-versa. Na Figura 7a são mostrados os dados obtidos para os filmes preparados a $100^{\circ} \mathrm{C}$. Estes apresentaram um comportamento constante durante os primeiros 100 pulsos, ou seja, a corrente em função do tempo permaneceu estável durante a ciclagem. Os filmes secos a $400^{\circ} \mathrm{C}$ apresentaram valores de corrente ligeiramente menores, mas sua estabilidade foi igual a dos filmes secos a $100^{\circ} \mathrm{C}$.
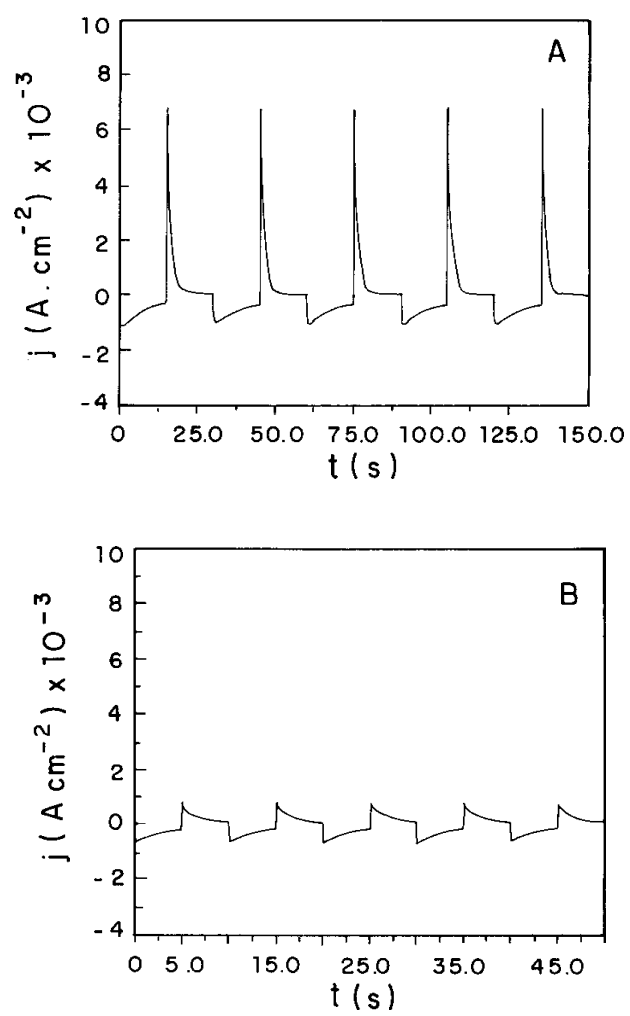

Figura 7. Cronoamperometria de filmes de $\mathrm{TiO}_{2}$ preparados a partir de sóis recém-preparados. Tprep $=50^{\circ} \mathrm{C}$ e Tsec $=100^{\circ} \mathrm{C}$. (a) Volume de $\mathrm{HCl}=50 \mathrm{~mL}$. (b) Volume de $\mathrm{HCl}=100 \mu \mathrm{L}$.

Os dados de cronoamperometria também permitiram obter uma estimativa do valor do coeficiente de difusão dos contra íons. Neste caso, foram construídos gráficos de carga (Q) em função da raiz quadrada do tempo $\left(\mathrm{t}^{1 / 2}\right)$. Novamente o coeficiente angular da porção reta dos gráficos construídos para o filme seco a $100^{\circ} \mathrm{C}$ (coeficiente angular $=0,060$ ) foi praticamente o dobro daquele observado para o filme seco a $400^{\circ} \mathrm{C}$ (coeficiente angular $=0,033$ ), evidenciando que o transporte de massa no primeiro é bem mais rápido.

Após a ciclagem do potencial, os filmes foram polarizados a diferentes potenciais e foram registrados espectros na região do ultravioleta-visível. Tanto para o $\mathrm{TiO}_{2}$ seco a $400^{\circ} \mathrm{C}$ como a $100^{\circ} \mathrm{C}$, mesmo após a ciclagem (100 pulsos), a variação de cor foi semelhante à observada na Figura 2.

$\mathrm{Na}$ Figura $7 \mathrm{~b}$ são mostrados os dados de cronoamperometria de filmes de $\mathrm{TiO}_{2}$ preparados usando-se um volume de $\mathrm{HCl}$ igual a $100 \mu \mathrm{L}$. Estes foram bem menos estáveis ao processo de ciclagem do potencial, pois foi observada uma queda significativa da corrente já nos primeiros 50 ciclos. Os filmes secos a $400^{\circ} \mathrm{C}$ foram ainda mais sensíveis, apresentando uma queda na corrente já nos primeiros 10 ciclos. Em ambos os casos, a 
variação de cor dos filmes também foi comprometida (os filmes praticamente não mudaram de cor com a polarização).

\section{Microscopia eletrônica de varredura}

As análises feitas por microscopia eletrônica de varredura, Figura 8, mostraram que os filmes de $\mathrm{TiO}_{2}$ apresentam morfologias diferentes dependendo da região do eletrodo observada. Nas regiões próximas das bordas do eletrodo, foi possível visualizar uma camada bastante trincada, cujos pedaços apresentam poros de diferentes dimensões. Para os filmes secos a $100^{\circ} \mathrm{C}$ o tamanho dos poros varia de aproximadamente 0,5 a $0,1 \mu \mathrm{m}$. Para os filmes secos a $400^{\circ} \mathrm{C}$ os poros parecem ter dimensões menores. Na região central dos eletrodos não foram visualizadas as trincas. Neste caso, o $\mathrm{TiO}_{2}$ seco a $100^{\circ} \mathrm{C}$ apresentou uma morfologia similar à de uma rede e para o $\mathrm{TiO}_{2}$ seco a $400^{\circ} \mathrm{C}$ a morfologia foi totalmente lisa e homogênea.
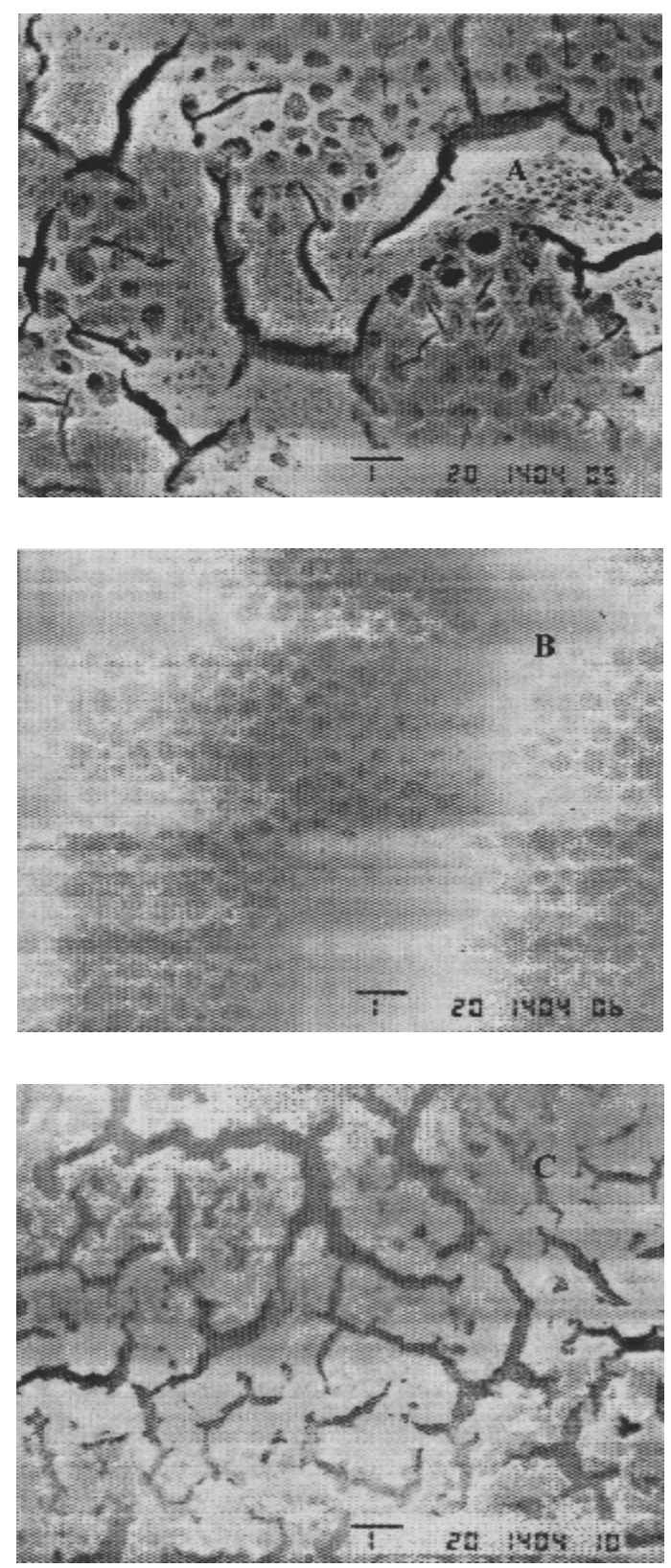

Figura 8. Microscopia eletrônica de varredura de filmes de $\mathrm{TiO}_{2}$ preparados a partir de sóis recém-preparados. Tprep $=50^{\circ} \mathrm{C}$ e volume de $\mathrm{HCl}=50 \mu \mathrm{L}$. (a, b) Tsec $=100^{\circ} \mathrm{C}$. (c) Tsec $=400^{\circ} \mathrm{C}$. (a, c) Bordas do eletrodo. (b) Região central do eletrodo.
Certamente, estas diferenças de morfologia influenciam as propriedades de transporte dos contra íons nos filmes. Conforme verificado anteriormente, o transporte de massa nos filmes preparados a $100^{\circ} \mathrm{C}$ foi mais rápido e provavelmente facilitado porque estes são bem menos compactos.

\section{Difratometria de raios-X}

A análise de difração de raios- $X$ foi feita para os filmes de $\mathrm{TiO}_{2}$ preparados a 100 e a $400^{\circ} \mathrm{C}$. Nestes casos, a temperatura de preparação dos sóis foi $50^{\circ} \mathrm{C}$ (sóis recém-preparados) e o volume de $\mathrm{HCl}$ usado foi $50 \mu \mathrm{L}$. Infelizmente, não foi possível obter informações sobre a estrutura do filme. Os difratogramas dos eletrodos contendo o $\mathrm{TiO}_{2}$ foram iguais ao do ITO limpo. Provavelmente a quantidade de sol depositada sobre o ITO foi insuficiente para se ter filmes com espessura adequada para tal análise. A mesma quantidade de sol tem sido depositada sobre placas de platina cuja área é a metade da área dos eletrodos de ITO usados aqui. Nestes casos, a análise por difração de raios- $\mathrm{X}$ tem mostrado que os filmes secos a $100^{\circ} \mathrm{C}$ são amorfos, os secos a 400 e a $600^{\circ} \mathrm{C}$ apresentam picos de difração similares ao $\mathrm{TiO}_{2}$ anatase, e os secos a $800^{\circ} \mathrm{C}$ similares ao $\mathrm{TiO}_{2}$ rutilo ${ }^{39}$. Pretende-se preparar novos filmes para confirmar se sobre o ITO o comportamento é similar ao observado sobre a platina, ou se o substrato pode estar influenciando de algum modo a estrutura do filme durante sua formação. Contudo, a temperatura usada na secagem do material deve ser no máximo igual a $400^{\circ} \mathrm{C}$, pois acima dessa temperatura, o ITO torna-se amarelado e mais resistivo.

\section{CONCLUSÃO}

Os resultados obtidos mostraram que as propriedades eletroquímicas e eletrocrômicas dos filmes de $\mathrm{TiO}_{2}$ são muito sensíveis às condições de preparação. Para obter filmes com propriedades eletrocrômicas satisfatórias, os sóis devem ser preparados a temperaturas superiores a $25^{\circ} \mathrm{C}$, deve-se usar um volume de ácido suficiente apenas para evitar a precipitação imediata do óxido (no caso, a mistura foi constituída de $30 \mathrm{~mL}$ de álcool, $1,2 \mathrm{~mL}$ de precursor e $50 \mu \mathrm{L}$ de $\mathrm{HCl}$ concentrado), e os sóis devem ser utilizados logo após a sua preparação (sóis recém-preparados). Nestas condições, os filmes apresentam também uma boa estabilidade frente a experimentos de cronoamperometria, nos quais o material é submetido a ciclos de oxidação e redução repetitivamente. A corrente de pico em função do tempo permaneceu estável durante 100 ciclos de oxidação e redução.

A temperatura usada na preparação dos filmes de $\mathrm{TiO}_{2}$ influencia sua morfologia. Filmes mais densos são obtidos quando a temperatura de secagem é aumentada de 100 para $400^{\circ} \mathrm{C}$. As propriedades de transporte dos contra íons intercalantes também são influenciadas. Estimativas do coeficiente de difusão dos íons obtidas a partir de experimentos de voltametria cíclica em diferentes velocidades de varredura e de cronoamperometria mostraram que nos filmes secos a $100^{\circ} \mathrm{C}$ o transporte de massa é duas vezes mais rápido que nos filmes secos a $400^{\circ} \mathrm{C}$. Em ambos os casos, $i_{p}$ variou linearmente com a raiz quadrada da velocidade de varredura. Tal comportamento sugere que o transporte de massa ocorre através de uma difusão linear semi-infinita.

\section{AGRADECIMENTOS}

Os autores agradecem à FAPESP (Proc. 95/9506-4, 96/69420 e $98 / 3920-1$ ).

\section{REFERÊNCIAS}

1. Wu, Y.; Hu, L.; Jiang, Z.; Ke, Q.; J. Electrochem. Soc. 1997, 144, 1728.

2. Kavan, L.; Gratzel, M.; Rathousky, J.; Zukal, A.; J. Electrochem. Soc. 1996, 143, 394. 
3. Hamasaki, Y.; Ohkubo, S.; Murakami, K.; Sei, H.; Nogami, G.; J. Electrochem. Soc. 1994, 141, 660.

4. Kokubu, T.; Nakahara, Y.; Yamane, M.; Aizawa, M.; J. Electroanal. Chem. 1993, 347, 123.

5. Liu, B.; Hu, R.; Deng, J.; Anal. Chim. Acta 1997, 341, 161.

6. Aylott, J. W.; Richardson, D. J.; Russell, D. A.; The Analyst 1997, 122, 77.

7. Lee, S. -K.; Okura, I.; The Analyst 1997, 122, 81.

8. Tavcar, G.; Kalcher, K.; Ogorevc, B.; The Analyst 1997, $122,371$.

9. Yang, S. -L.; Wu, J. -M.; J. Mater. Sci. 1991, 26, 631.

10. Bouting, P.; Mugnier, J.; Valeur, B.; J. Fluorescence 1997, 7, 215s.

11. Alves, V. A.; da Silva, L. A.; Boodts, J. F. C.; J. Chem. Soc. 1998, 94, 711.

12. Guglielmi, M.; Colombo, P.; Batteglin, G.; J. Electrochem. Soc. 1992, 139, 1655.

13. Davies, A.; Hobson, R. J.; Hudson, M. J.; Macklin, W. J.; Neat, R. J.; J. Mater. Chem. 1996, 6, 49.

14. Marci, G.; Palmisano, L.; Sclafani, A.; Venezia, A.M.; Campostrini, R.; Carturan, G.; Martin, C.; Rives, V.; Solana, G.; J. Chem. Soc., Faraday Trans. 1996, 92, 819.

15. Maksimov, Y. V.; Suzdalev, I. P.; Tsodikov, M. V.; Kugel, V. Y.; Bukhtenko, O. V.; Slivinsky, E. V.; Navio, J. A.; J. Mol. Catal. A: Chem. 1996, 105, 167.

16. Vinodgopal, K.; Hotchandani, S.; Kamat, P. V.; J. Phys. Chem. 1993, 97, 9040.

17. Lopez, T.; Tzompantzi, F.; Novaro, O.; J. Catal. 1999, $181,285$.

18. Zhan, Z.; Zeng, H. C.; J. Non-Cryst. Solids 1999, 243, 26.

19. Garbassi, F.; Balducci, L.; Ungarelli, R.; J. Non-Cryst. Solids 1998, 223, 190.

20. Ebener, S.; Winter, W.; J. Europ. Ceram. Soc. 1996, 16, 1179.

21. Andrianainarivelo, M.; Corriu, R.; Vioux, A.; J. Mater. Chem. 1996, 10, 1665.
22. Qunyin, Q.; Anderson, M. A.; J. Am. Ceram. Soc. 1993, 76, 2093.

23. Sharygin, L. M., Malykh, T. G.; Galkin, V. M.; Inorg. Mater. 1989, 25, 1284.

24. Signoretto, M.; Scarpa, M.; Benedetti, A.; J. Non-Cryst. Solids 1998, 225, 178.

25. Saha A.; Agrawal, D. C.; J. Mater. Sci. Lett. 1998, 17, 1333.

26. Rivas, P. C.; Caracoche, M. C.; Sanctis, O.; J. Mater. Res. 1997, 12, 493.

27. Lee, J. S.; Matsubars, T.; Tsuchiya, T.; J. Mater. Sci. 1997, 32, 5249.

28. Caruso, R.; Benavidez, E.; Serquis, A.; J. Mater. Res. 1997, 12, 2594

29. Rigden, J. S.; Walters, J. K.; Newport, R. J.; J. Phys. Condensed Matter 1997, 9, 4001.

30. Rigden, J. S.; Newport, R. J.; Bushnell-Wye, G.; J. Mater. Chem. 1996, 6, 337.

31. Alves, V. A.; da Silva, L. A.; Boodts, J. F. C.; Electrochim. Acta 1998, 44, 1525.

32. Keomany, D.; Petit, J. P.; Deroo, D.; Solar Energy Mater. Solar Cells 1995, 36, 397.

33. Stangar, U. L.; Orel, B.; Ogorevc, B.; Acta Chim. Slov. 1994, 41, 39.

34. Alves, V. A.; da Silva, L. A.; Boodts, J. F. C.; Electrochim. Acta 1997, 42, 271.

35. Kameyama, K.; Shohje, S.; Onoue, S.; J. Electrochem. Soc. 1993, 140, 1024.

36. Bange, K.; Gambke, T.; Adv. Mater. 1990, 2, 10.

37. Nabavi, M.; Doeuff, S.; Sanchez, C.; Livage, J.; Mater. Sci. Eng. 1989, B3, 203.

38. Livage, J.; Henry, M.; Sanchez, C.; Prog. Solid St. Chem. 1988, 18, 259.

39. Transferetti, B. C.; Davazano, C. U.; Zoppi, R. A.; da Cruz, N. C.; de Moraes, M. A. B.; Phys. Rev. 2000, submetido. 